\title{
New insights into genotype-phenotype correlation for GLI3 mutations
}

\author{
Florence Démurger ${ }^{1}$, Amale Ichkou ${ }^{2}$, Soumaya Mougou-Zerelli ${ }^{3,4}$, Martine Le Merrer ${ }^{3}$, Géraldine Goudefroye ${ }^{2}$, \\ Anne-Lise Delezoide ${ }^{5}$, Chloé Quélin ${ }^{1}$, Sylvie Manouvrier ${ }^{6}$, Geneviève Baujat ${ }^{2,3,7}$, Mélanie Fradin ${ }^{1}$, \\ Laurent Pasquier ${ }^{1}$, André Megarbané ${ }^{8}$, Laurence Faivre ${ }^{9}$, Clarisse Baumann ${ }^{10}$, Sheela Nampoothiri ${ }^{11}$, \\ Joëlle Roume ${ }^{12}$, Bertrand Isidor ${ }^{13}$, Didier Lacombe ${ }^{14}$, Marie-Ange Delrue ${ }^{14}$, Sandra Mercier ${ }^{13}$, Nicole Philip ${ }^{15}$, \\ Elise Schaefer ${ }^{16}$, Muriel Holder ${ }^{6}$, Amanda Krause $^{17}$, Fanny Laffargue ${ }^{18}$, Martine Sinico ${ }^{19}$, Daniel Amram ${ }^{20}$, \\ Gwenaelle Andrée ${ }^{21}$, Alain Liquier ${ }^{22}$, Massimiliano Rossi ${ }^{23}$, Jeanne Amiel ${ }^{2,3,7}$, Fabienne Giuliano ${ }^{24}$, \\ Odile Boute $^{6}$, Anne Dieux-Coeslier ${ }^{6}$, Marie-Line Jacquemont ${ }^{25}$, Alexandra Afenjar ${ }^{26,27}$, Lionel Van Maldergem ${ }^{28}$, \\ Marylin Lackmy-Port-Lis ${ }^{29}$, Catherine Vincent- Delorme ${ }^{30}$, Marie-Liesse Chauvet $^{2,3}$, Valérie Cormier-Daire ${ }^{2,3,7}$, \\ Louise Devisme ${ }^{31}$, David Geneviève ${ }^{32}$, Arnold Munnich ${ }^{2,3,7}$, Géraldine $\operatorname{Viot}^{33}$, Odile Raoul ${ }^{2}$, Serge Romana ${ }^{2,3,7}$, \\ Marie Gonzales $^{34}$, Ferechte Encha-Razavi ${ }^{2,7}$, Sylvie Odent ${ }^{1}$, Michel Vekemans ${ }^{2,3,7}$ and Tania Attie-Bitach ${ }^{\star 2,3,7}$
}

The phenotypic spectrum of GL/3 mutations includes autosomal dominant Greig cephalopolysyndactyly syndrome (GCPS) and Pallister-Hall syndrome (PHS). PHS was first described as a lethal condition associating hypothalamic hamartoma, postaxial or central polydactyly, anal atresia and bifid epiglottis. Typical GCPS combines polysyndactyly of hands and feet and craniofacial features. Genotype-phenotype correlations have been found both for the location and the nature of GLI3 mutations, highlighting the bifunctional nature of GLI3 during development. Here we report on the molecular and clinical study of 76 cases from 55 families with either a GLI3 mutation (49 GCPS and 21 PHS), or a large deletion encompassing the GLI3 gene (6 GCPS cases). Most of mutations are novel and consistent with the previously reported genotype-phenotype correlation. Our results also show a correlation between the location of the mutation and abnormal corpus callosum observed in some patients with GCPS. Fetal PHS observations emphasize on the possible lethality of GLI3 mutations and extend the phenotypic spectrum of malformations such as agnathia and reductional limbs defects. GLI3 expression studied by in situ hybridization during human development confirms its early expression in target tissues.

European Journal of Human Genetics (2015) 23, 92-102; doi:10.1038/ejhg.2014.62; published online 16 April 2014

\section{INTRODUCTION}

Mutations in the GLI3 gene lead to several clinical phenotypes including Greig cephalopolysyndactyly syndrome (GCPS; MIM\# 175700) $)^{1}$ and Pallister-Hall syndrome (PHS; MIM\# 146510). ${ }^{2}$ PHS first described in 1980 by Hall as a lethal condition in neonatal period $^{3,4}$ associates mainly hypothalamic hamartoma $(\mathrm{HH})$, postaxial polydactyly (PD), bifid epiglottis and imperforate anus (IA). PHS forms a spectrum from very mild cases with subtle insertional PD to severe cases. ${ }^{5}$ Typical GCPS is characterized by polysyndactyly in hands and/or feet, craniofacial abnormalities, such as macrocephaly and hypertelorism, ${ }^{6}$ and developmental delay in individuals with large deletions encompassing GLI3.?

GCPS and PHS are distinct entities both caused by mutations in the transcription factor GLI3, a modulator of Sonic hedgehog (SHH) pathway with a bifunctional nature, either activator or repressor. In the presence of SHH, full-length GLI3 functions as a transcriptional activator (GLI3A), whereas in the absence of SHH, GLI3 is cleaved to produce a repressor (GLI3R). ${ }^{8}$

\footnotetext{
${ }^{1}$ Service de Génétique Clinique, CLAD-Ouest, Hôpital Sud, Rennes, France; ²Département de Génétique, Hôpital Necker-Enfants Malades, Assistance Publique -Hôpitaux de Paris (AP-HP), Paris, France; ${ }^{3}$ Inserm U1163, Hôpital Necker-Enfants Malades, Paris, France; ${ }^{4}$ Service de Cytogénétique et Biologie de la Reproduction, CHU Farhat Hached, Sousse, Tunisia; ${ }^{5}$ Service de Fœtopathologie, Hôpital Robert Debré, AP-HP, Paris, France; ${ }^{6}$ Service de Génétique Clinique, CLAD-NdF, CHRU de Lille, Lille, France; ${ }^{7}$ Université Paris Descartes - Sorbonne Paris Cité, Institut Imagine, Paris, France; ${ }^{8}$ Unité de Génétique Médicale, Faculté de Médecine, Université St Joseph, Beirut, Lebanon; ${ }^{9}$ Centre de Génétique, Hôpital d'enfants, CHU de Dijon, Dijon, France; ${ }^{10}$ Département de Génétique, Hôpital Robert Debré, AP-HP, Paris, France; ${ }^{11}$ Department of Pediatric Genetics, Amrita Institute of Medical Sciences, Kerala, India; ${ }^{12}$ Unité de Génétique Médicale, CH Poissy St-Germain-en-Laye, Poissy, France; ${ }^{13}$ Service de Génétique Médicale, Unité de Génétique Clinique, CLAD-Ouest, CHU de Nantes, Nantes, France; ${ }^{14}$ Service de Génétique Médicale, CHU de Bordeaux, Bordeaux, France; ${ }^{15}$ Département de Génétique Médicale, Hôpital d’Enfants de La Timone, Marseille, France; ${ }^{16}$ Service de Génétique Médicale, CHU de Strasbourg, Strasbourg, France; ${ }^{17}$ Division de Génétique Humaine, Hospital St Hillbrow, Johannesburg, South Africa; 18 Service de Génétique Médicale, CHU Estaing, Clermont-Ferrand, France; ${ }^{19}$ Service d'Anatomie Pathologique, CH Intercommunal de Créteil, Créteil, France; ${ }^{20}$ Unité de Génétique Clinique, $\mathrm{CH}$ Intercommunal de Créteil, Créteil, France; ${ }^{21}$ Service d’Anatomie Pathologique, CHU Pellegrin, Bordeaux, France; ${ }^{22}$ Laboratoire de Cytogénétique Bioffice, Bordeaux, France; ${ }^{23}$ Service de Génétique, Hospices Civils de Lyon, CHU de Lyon, France; ${ }^{24}$ Service de Génétique Médicale, Hôpital de l'Archet II, CHU de Nice, France; ${ }^{25}$ Unité de Génétique Médicale, CHU la Réunion, France; ${ }^{26}$ Service de Génétique, Hôpital Pitié Salpêtrière, Paris, France; ${ }^{27}$ Centre de Référence des Malformations et Maladies Congénitales du Cervelet, Hôpital Trousseau, AP-HP, Paris, France; ${ }^{28}$ Centre de Génétique Humaine, Université de Franche-Comté, Besançon, France; ${ }^{29}$ Unité de Génétique Clinique, $\mathrm{CHU}$ de Pointe à Pitre, France; ${ }^{30}$ Service de Génétique, $\mathrm{CH}$ d’Arras, France; ${ }^{31}$ Institut de Pathologie, Centre de Biologie-Pathologie, CHRU de Lille, France; ${ }^{32}$ Département de Génétique Médicale, CHU de Montpellier, France; ${ }^{33}$ Unité de Génétique, Maternité Port-Royal, Hôpital Cochin, AP-HP, Paris, France; ${ }^{34}$ Service de Génétique et d’Embryologie Médicales, Hôpital Armand Trousseau, AP-HP, Paris, France

*Correspondence: Professor T Attie-Bitach, Département de Génétique et INSERM U781 Hôpital, Necker-Enfants Malades 149 rue de Sèvres, Paris 75743 , Cedex 15, France. Tel: +33 (0) 144495144; Fax: +33 (0) 171196420; E-mail: tania.attie@inserm.fr
}

Received 27 October 2013; revised 20 January 2014; accepted 13 March 2014; published online 16 April 2014 
Previous reports demonstrate a robust genotype-phenotype correlation of GLI3 mutations. ${ }^{9,10}$ Truncating mutations in the middle third of the gene generally cause PHS, resulting in a constitutive repressor protein. By contrast, haploinsufficiency resulting from chromosomal rearrangements, but also missense, splicing or truncating mutations elsewhere in the gene cause GCPS by loss of the DNA-binding capacity $^{11}$ or activation of nonsense-mediated mRNA decay, ${ }^{12}$ or by the formation of an unstable or mislocalized protein. ${ }^{13,14}$

In this study, we report on the clinical and molecular data of a French cohort of 76 individuals from 55 families carrying a GLI3 molecular defect. Most of mutations are novel and consistent with the previously reported genotype-phenotype correlation. In addition, our results also show a correlation between the location of the mutation and corpus callosum dygenesis observed in some GCPS individuals. Fetal observations emphasize on the possible lethality of GLI3 mutations, extend the phenotypic spectrum of malformations to severe craniofacial and reductional limb defects. GLI3 expression studied by in situ hybridization during human development confirms its early expression in target tissues including in pharyngeal arches, and later in mandible.

\section{PATIENTS AND METHODS}

\section{Patients}

Index cases were tested for mutations in the GLI3 gene because of the presence of clinical findings compatible with the diagnosis of GCPS or PHS and 76 cases from 55 families are included in this upon identification of a GLI3 molecular defect.

A total of 55 patients from 38 families with features compatible with GCPS (polysyndactyly in hands and/or in feet and/or dysmorphic features associating high forehead, macrocephaly or widely spaced eyes and/or corpus callosum anomalies) were identified with a GLI3 mutation or rearrangement, 39 cases were familial and 16 sporadic.

Also, 21 PHS individuals from 17 families with postaxial or insertional PD and/or HH with pathogenic GLI3 mutations have been included. Among them, 13 were sporadic, 7 familial and 1 of unknown inheritance. Antenatal cases were selected for either $\mathrm{HH}$ or IA plus at least $2 / 5$ features belonging to the PHS spectrum namely intrauterine growth retardation (IUGR), limb malformation, heart disease, micropenis and renal anomaly. In all seven fetuses with GLI3 mutation, pregnancy was terminated because of severe clinical findings, in accordance with French legislation. A written informed consent for genetic analysis was obtained from each family before testing, and for autopsy in all fetal cases.

\section{Molecular genetic studies}

GLI3 mutation screening. Genomic DNA was extracted from frozen fetal tissue or amniocytes for the fetal cases and from peripheral blood samples in the postnatal cases. The 14 coding exons and the adjacent intronic regions of the GLI3 gene were amplified using GLI3-specific primers pairs (available on request). Direct sequencing of PCR products was performed using the Big Dye Terminator Cycle Sequencing Kit v3 (Applied Biosystems, Courtaboeuf, France) and analyzed on an ABI3130 automated sequencer (Applied Biosystems). Sequences were analyzed with Seqscape software v2.5 (Applied Biosystems). Sequence data were compared with the GLI3 reference sequence NM_000168.5, and mutation named according to the HGVS nomenclature and checked by the Mutalyzer programme. ${ }^{15}$ Mutations have been submitted to the public database LOVD.

All missense variants identified were investigated by in silico analysis using SIFT and PolyPhen2. Parental studies were done in sporadic cases to confirm a de novo occurrence of the alterations when parental DNA was available. We classified novel variants as pathogenic mutations the nonsense, frameshift and splice variants, and the missense variant, which affected conserved nucleotide or amino acid, segregating with the disease in familial cases, and/or apparently de novo in sporadic cases. Confirmation of biological parentage was performed for the de novo missense mutation only.

GLI3 rearrangement analysis. Individuals without GLI3 coding sequence mutations underwent fluorescent in situ hybridization (FISH), Multiplex
Ligation-dependent Probe Amplification (SALSA MLPA KIT P179 Limb Malformations-1-MRC-Holland, Amsterdam, The Netherlands) or Array comparative genomic hybridization (Array CGH Agilent 244 or $180 \mathrm{~K}$ oligonucleotide microarrays, Agilent Technologies, Santa Clara, CA, USA).

\section{Gene expression analyses using in situ hybridization}

Human embryos and fetal tissues were obtained from legally terminated pregnancies in agreement with French law (94-654 of 29 July 1994), following National Ethics Committee recommendations and with approval from the Necker Hospital ethics committee. Five developmental stages using Carnegie staging $(\mathrm{CS})^{16}$ were studied: C14, C16, C18, C19 and 8.5 weeks of development. Tissues were fixed in $4 \%$ phosphatase-buffered paraformaldheyde, dehydrated and embedded in paraffin blocks, and $5 \mu \mathrm{m}$-thick serial sections were cut. Exon 3 primers were selected for PCR amplification (3F-TACTTCTTTTCCGGGAGAGG and 3R-CCATAGCTC CTGAACAAGTG). Sense and antisense riboprobes were generated using either T7 or T3 RNA polymerase. Riboprobe labeling, tissue fixation, hybridization and developing were carried out according to standard protocols as described previously. ${ }^{17}$ No hybridization signal was detected with the labeled sense probe, confirming that the expression pattern obtained with the antisense probe was specific. Adjacent slides were hematoxylin/eosin stained for morphological studies.

\section{RESULTS}

Detailed clinical phenotypes and molecular results are described in Table 1 (GCPS) and Table 2 (PHS). Frequencies reported in Tables 3 and 4 are represented as the ratio between the number of patients with a particular finding and the total number of patients for which the information was available.

\section{GLI3 mutations and deletions}

Greig cephalopolysyndactyly syndrome. We identified 32 causative mutations and 6 large deletions in 38 GCPS index cases. Among the 16 sporadic cases, the de novo occurrence in the proband was confirmed for 6 patients after analysis of both parents. Three mutations were recurrent (c.1874G $>$ A, c.4463del and c.444C $>$ A identified each in two families). Overall, $8 / 38$ (21\%) were nonsense mutations, $17 / 38(45 \%)$ were frameshift mutations predicting a premature stop codon, $6 / 38(16 \%)$ were missense mutations, $1 / 38$ (3\%) was a splice mutation and 6/38 (16\%) were complete deletions of the gene. Nine of them were located in the N-terminal part of GLI3 before the zinc-finger domain predicting a prematurely terminated protein lacking the DNA-binding domain. Interestingly, all 6 missense mutations were within DNA-binding domain extending from aminoacid 462 to 645 encoded by exons 10 to 13 of the GLI3 gene (Figure 1). All six missense mutations are predicted to be probably damaging to the protein function in silico by both PolyPhen-2 and SIFT softwares, involving conserved amino acids. Ten truncating mutations are located in the $1 / 3$ end of the protein, within the transactivation domains TA2 and TA1. ${ }^{11}$ Interestingly, two truncating mutations (c.2082_2083delinsAGAGAAGCC and c.3427_3443del) were in the previously defined PHS region (between cDNA positions 1998 and 3481). ${ }^{9}$ Among the 29 different mutations found in this series, two (c.868C $>\mathrm{T}$ and c.1874G $>\mathrm{A}$ ) were previously described in other patients ${ }^{9}$ and 27 are novel mutations. Of note, a frameshift mutation (c.1543_1544dup) found in two affected sibs, was present at low level in DNA extracted from blood of their father (Family G068), suggesting a somatic mosaicism. Along the same line, a FISH analysis revealed a GLI3 deletion in only $56 \%$ of blood cells of a patient (G059) with bilateral preaxial PD of the feet and developmental delay. At least two patients (G005 and G019) had Greig cephalopolysyndactyly contiguous gene syndrome (GCPS-CGS) 


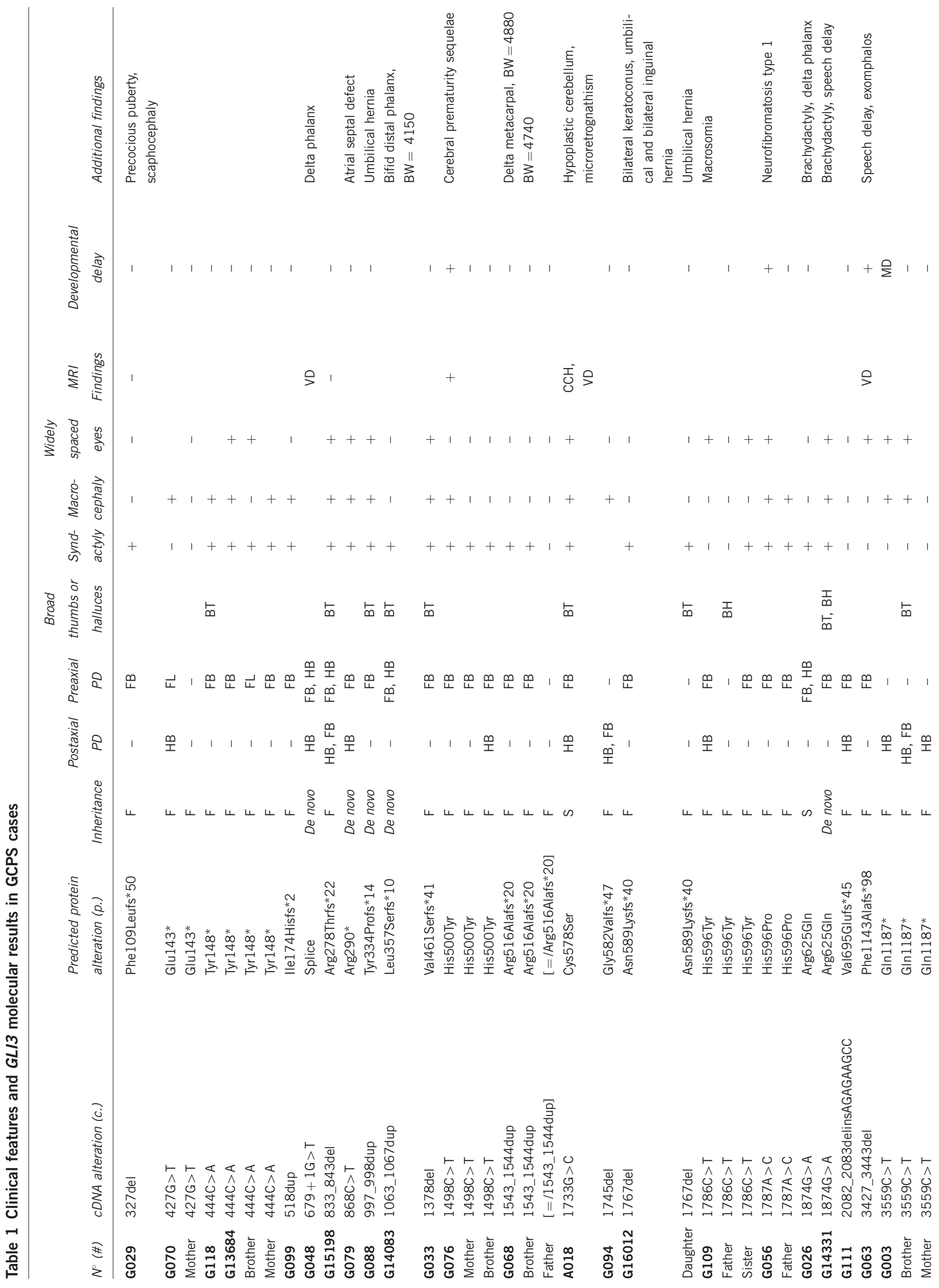




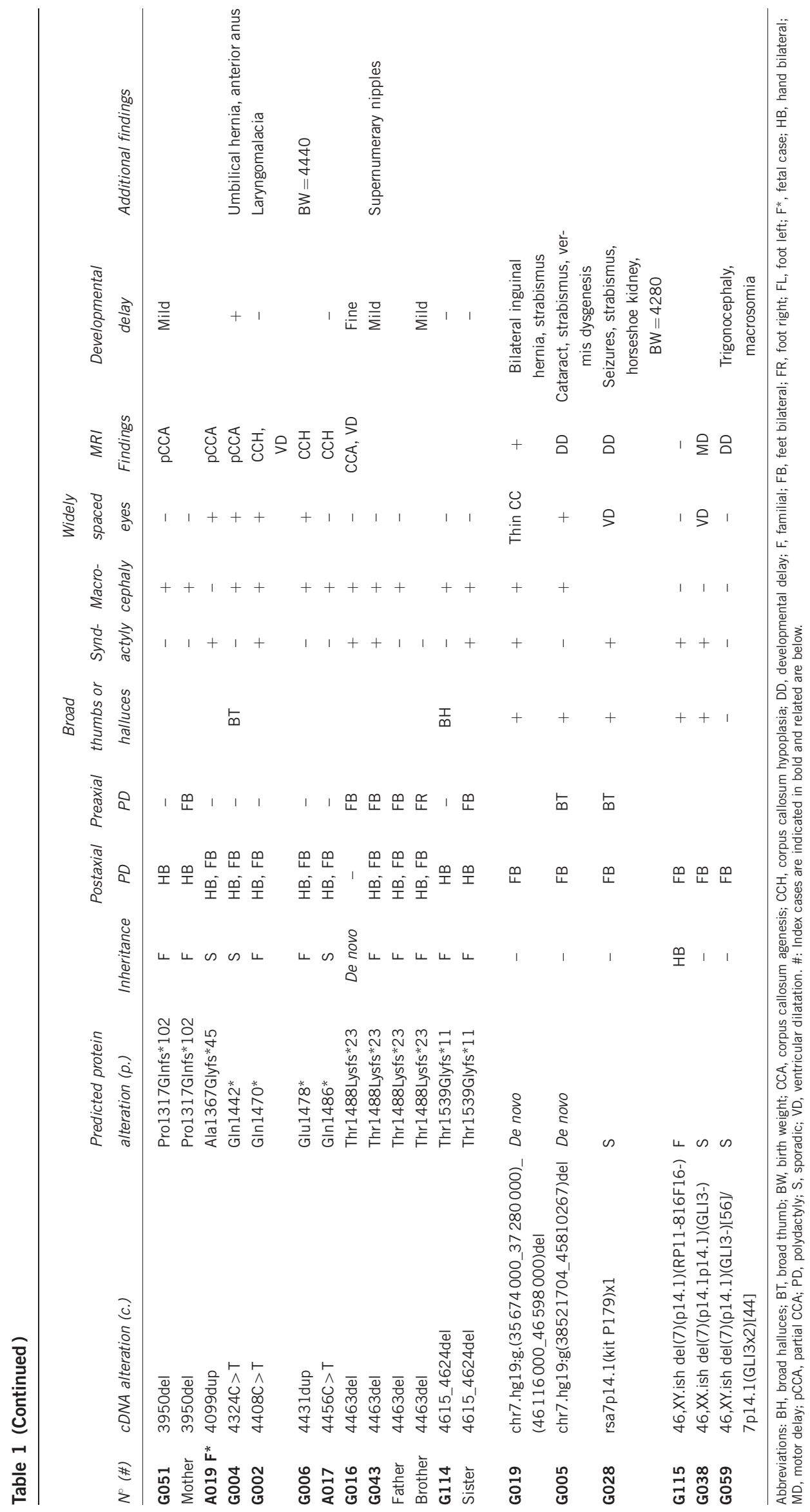




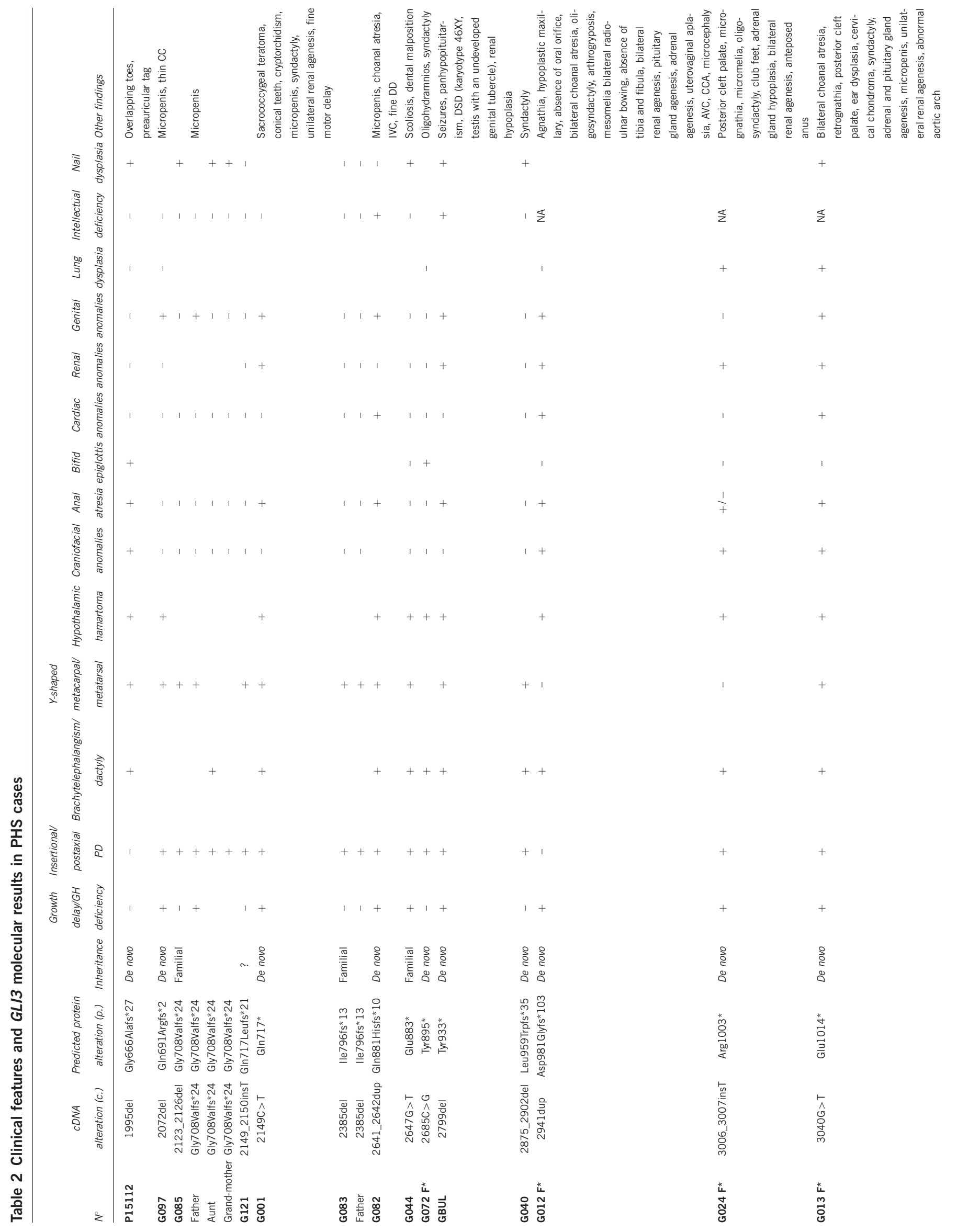


Table 3 Frequencies of clinical features in GCPS individuals

Features

Frequency

Facial anomalies

Widely spaced eyes

$43 \%(20 / 47)$

Macrocephaly

$60 \%(32 / 53)$

Craniosynostosis

$4 \%(2 / 55)$

Hand anomalies

Preaxial polydactyly

$7 \%(4 / 55)$

Postaxial polydactyly

$45 \%(25 / 55)$

Broad thumbs

$22 \%(12 / 55)$

Foot anomalies

Preaxial polydactyly

$73 \%(40 / 55)$

Postaxial polydactyly

$20 \%(11 / 55)$

Syndactyly

$64 \%(34 / 53)$

Cerebral anomalies

Corpus callosum anomalies

$50 \%(9 / 18)$

Ventricular dilatation

$39 \%(7 / 18)$

Developmental delay

Severe

$11 \%(5 / 45)$

Mild

$20 \%(9 / 45)$

Birth weight $>4000 \mathrm{~g}$

$12 \%(7 / 55)$

Inguinal or umbilical hernia

caused by haploinsufficiency of GLI3 and adjacent genes confirmed by array-CGH with a deletion of 7 and $9 \mathrm{Mb}$, respectively. Both presented preaxial PD of the feet, developmental delay and ophthalmologic findings (strabismus, cataract). The mutations segregated with the disease in all familial cases. In one apparently sporadic case (G070), the mutation was inherited from a healthy mother with no sign of GCPS.

Pallister-Hall syndrome. We identified heterozygous GLI3 mutations in 21 patients from 17 families with features of PHS ( $\mathrm{HH}$ and/or insertional or postaxial PD and/or Y-shaped metacarpal) and all were truncating (12 frameshift and 5 nonsense). Among the 13 sporadic cases, the de novo occurrence was confirmed in 13. Three mutations were previously reported in other patients (c.2149C > T, c.3040G $>$ T and c.3386_3387del $)^{9,18}$ and 14 mutations were novel. All mutations identified in probands with PHS were $3^{\prime}$ of the DNA-binding domain and predicted the formation of a truncated protein. All were located in the previously described PHS region stretching from aa 667 to 1161, one starting just one amino-acid upstream, which predicts a premature termination codon 27 triplets downstream (P15112). All mutations found in fetuses with severe phenotypes affect a delineated region of the middle third of GLI3 in the transactivation/CBP-binding region (Figure 1).

\section{Clinical and radiological findings}

GCPS

Limb anomalies. Preaxial PD of the feet was the most frequent finding (40/55) (Figures 2a, b and d), but broad halluces were also observed (Figure 2c). Complete preaxial PD of the hands was seen only in 1 case (Figure 2f) and broad thumbs in 12 cases (Figure $2 \mathrm{~g}$ ) with the presence in 2 cases of a delta phalanx or a bifid terminal phalanx on X-rays (Figure 2e). Postaxial PD was observed in 25/55 cases $(45 \%)$ in feet $(20 \%)$ or hands $(45 \%)$. The severity of the PD 
Table 4 Frequencies of clinical features in PHS individuals

\begin{tabular}{lc}
\hline Clinical features & Frequency \\
\hline Growth delay/GH deficiency & $53 \%(10 / 19)$ \\
Limb anomalies & \\
Y-shaped metacarpal & $83 \%(15 / 18$ \\
Postaxial polydactyly & $48 \%(10 / 21)$ \\
Insertional polydactyly & $48 \%(10 / 21)$ \\
Preaxial polydactyly & $0 \%(0 / 21)$ \\
Oligodactyly & $14 \%(3 / 21)$ \\
Syndactyly & $38 \%(8 / 21)$ \\
Brachydactyly/brachytelephalangism & $52 \%(13 / 21)$ \\
Mesomelia & $19 \%(4 / 21)$ \\
Nail hypoplasia & $69 \%(9 / 13)$ \\
Overlapping toes & $9 \%(2 / 21)$ \\
Cerebral anomalies & \\
Hypothalamic hamartoma & $100 \%(12 / 12)$ \\
Corpus callosum anomalies & $17 \%(2 / 12)$ \\
Craniofacial anomalies & \\
Micro/retro/agnathia & $24 \%(5 / 21)$ \\
Choanal atresia & $14 \%(3 / 21)$ \\
Cleft palate & $14 \%(3 / 21)$ \\
Chondroma & $9 \%(2 / 21)$ \\
Bifid epiglottis & \\
Anal anomalies & $44 \%(4 / 9)$ \\
Anal imperforation & \\
Anteposed anus & \\
Cardiac anomalies & $43 \%(9 / 21)$ \\
Interauricular communication & $5 \%(1 / 21)$ \\
Interventricular communication & \\
Atrioventricular communication & $9 \%(2 / 21)$ \\
Renal anomalies & $9 \%(2 / 21)$ \\
Genital anomalies & $5 \%(1 / 21)$ \\
Developmental delay & $5 \%(1 / 21)$ \\
Seizures & $41 \%(7 / 17)$ \\
\hline & $48 \%(10 / 21)$ \\
\hline & $50 \%(4 / 8)$ \\
& $21 \%(3 / 14)$ \\
\hline & $13 \%(2 / 15)$ \\
\hline
\end{tabular}

extended from a pedunculated postminimus (Figure $2 \mathrm{~h}$ ) to a fully formed supernumerary digit (Figure 2f). Syndactyly present in $64 \%$ $(34 / 53)$ may occur in any limb and varied from partial to complete cutaneous syndactyly of the digits. Metacarpals were not affected except the first metacarpal, sometimes shorter and squatter (Figure 2b).

Craniofacial dysmorphism. In our series, $43 \%$ of patients had widely spaced eyes and $60 \%$ had macrocephaly. Scaphocephaly and trigonocephaly were noted both in one case.

Cerebral anomalies. A brain MRI was performed in 18 patients. A ventricular dilatation was found in seven cases. Surprisingly, corpus callosum dysgenesis (hypoplasia or agenesis) were not found in patients with large deletion except one but mainly in those bearing a truncating mutation in the C-terminal region of GLI3 (7/9). In one family (G006), corpus callosum abnormalities were present in all affected individuals. Hypoplastic cerebellum was found in two patients (G005, A018) without molar tooth sign. Among patients with a large deletion encompassing GLI3, 5/6 manifested developmental delay and 4 had abnormal brain MRI findings. Apart from these deleted cases, a mild developmental delay (fine motor delay) was observed in nine cases. Among them, seven had a C-terminal mutation, the two others had neurofibromatosis type 1 and prematurity complications.

Other occasional signs. Other less common anomalies in GCPS included umbilical and inguinal hernias (six patients). Birth weight was indicated for 15 patients and macrosomia was noted for 7 .

Pallister-Hall syndrome

Limb anomalies. Limb anomalies were present in all 21 PHS individuals of our cohort. The most common feature was postaxial $(48 \%)$ or insertional PD (48\%) (Figures 3a and c). No patient with preaxial PD was recorded. Interestingly, Y-shaped metacarpal/metatarsal was visualized on X-rays in $83 \%$ of cases and in all other cases, numeric or morphologic anomalies of metacarpal/metatarsal were noted (Figures $3 \mathrm{c}$ and $\mathrm{d}$ ). Only one patient (P15112) had bilateral and symmetrical Y-shaped metacarpals without PD (Figure 3d). Brachydactyly with brachytelephalangism was observed in at least $52 \%$ of PHS cases and nail hypoplasia in $69 \%$.

Syndactyly $(38 \%)$ and overlapping toes $(9 \%)$ were frequently reported. Four fetuses with severe phenotypes exhibited mesomelia or micromelia and three of them presented oligodactyly, club feet and arthrogryposis (Figures 3b, g-j).

Neurological findings. A HH was present in 12 patients, all with mutations falling in the 'PHS' domain (Figures $3 \mathrm{e}$ and $\mathrm{f}$ ). In one fetus, neuropathological examination of the hypothalamic region found histological lesions of hamartoma, although a macroscopic mass was not visualized in the infandibular region (G024). Two cases displayed corpus callosum dysgenesis. Most patients had a normal intellectual efficiency, only three were slightly delayed. Seizures were reported in two cases as gelastic epilepsy.

Other findings. IUGR was found in $4 / 5$ fetuses and growth was delayed in 6 patients. Besides, the endocrine manifestations of a $\mathrm{HH}$ ranged from isolated growth hormone deficiency (4/13) to panhypopituitarism (1 case); 4/5 fetuses displayed adrenal hypoplasia. Oral anomalies were reported in all prenatal cases: cleft palate in three fetuses, micro/retrognatia in four and unexpectedly, a complete agnathia with absence of oral orifice in one (Figure 3c). Laryngeal examination revealed bifid epiglottis in half cases, always asymptomatic in postnatal cases. Choanal atresia was present in three patients and two displayed cervical or preauricular chondroma. Moreover, imperforate or anteposed anus was present in half PHS cases including all fetuses. Congenital heart defects were diagnosed in six patients: interauricular septal defect in two, interventricular communication in two, atrioventricular septal defect in one and an aortic arch anomaly in one. Renal anomalies were present in $41 \%$ ranging from kidney hypoplasia to agenesis and resulting in oligoamnios in prenatal cases with bilateral agenesis (three cases). Genitourinary anomalies including micropenis (nine cases), hypospadias (one case) and uterovaginal aplasia (one case) were present in half of the individuals. A severe developmental sexual disorder was present in a girl with a male caryotype exhibiting an undeveloped genital tubercle. Lung anomalies including abnormal lobulation or hypoplatic lungs were present in four individuals.

\section{In situ hybridization of GLI3}

GLI3 expression pattern was studied during early human development using in situ hybridization on human embryo sections at CSs 14 (day 32), 16 (day 40), 18 (day 44), and 19 (day 47) and at 8.5 weeks of 


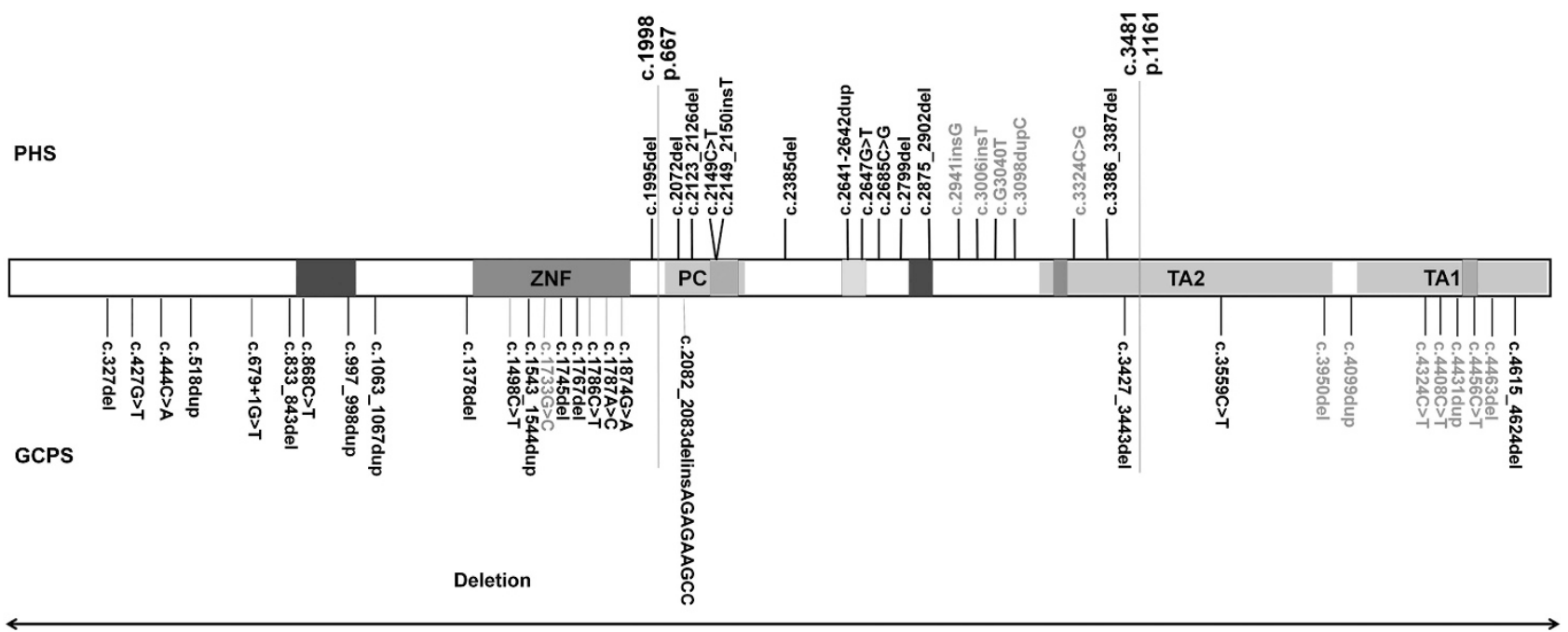

Figure 1 Schematic representation of GLI3 domains and localization of the GLI3 mutations reported in this study. Red bars at the nucleotides 1998 and 3481 divide the gene into three segments, limiting the PHS region as described elsewhere. ${ }^{9}$ The colored boxes within GLI3 represent the seven regions of similarity between human GLI proteins originally defined by Ruppert et al. ${ }^{34}$ ZNF: zinc-finger domain (aa 462-645), PC: proteolytic cleavage site, TA1 (aa 1376-1580) and TA2 (aa 1044-1322): two independent transactivation domains as described by Kalff-Suske et al. ${ }^{11}$ Mutations written in red: PHS patients with severe phenotypes; in green: GCPS cases with abnormal corpus callosum; black bars: truncating nonsense and frameshift mutations; purple bar: splice mutation; blue bars: missense mutations. A full color version of this figure is available at the European Journal of Human Genetics journal online.

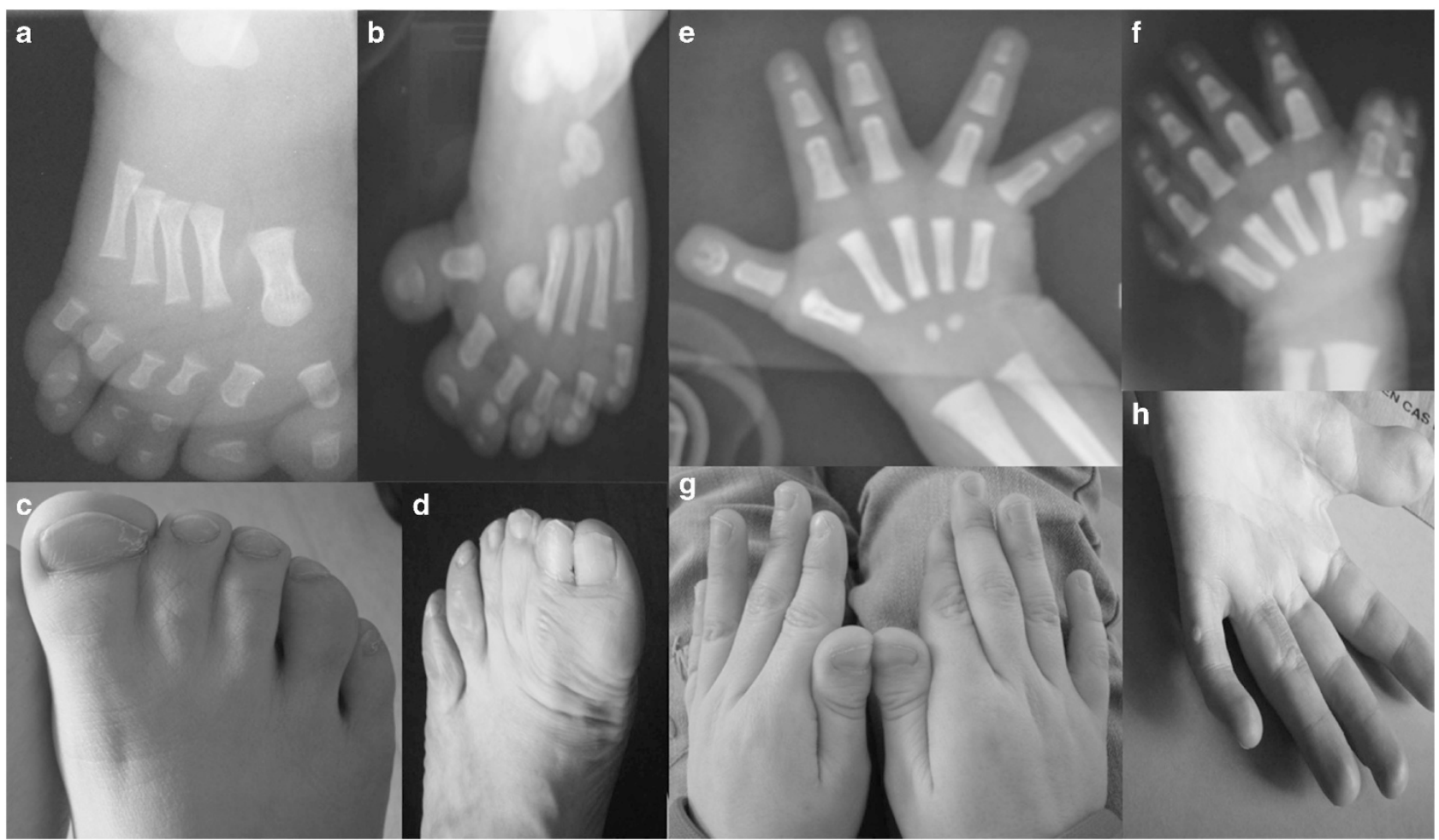

Figure 2 Photographs and radiographies of GCPS cases with identified GL/3 mutation. (a, b) Preaxial polysyndactyly in the feet with a broad first metacarpal on X-rays (G076, G14083). (c) Broad hallux and syndactyly (G16012, daughter). (d) Preaxial polysyndactyly (G16012). (e) Bifid terminal phalanx of the thumb (G14083). (f) Heptadactyly (preaxial and postaxial PD, G15198). (g) Broad thumbs (G16012, daughter). (h) Broad thumb and postaxial PD type (b) (G16012).

development. At day 32, GLI3 was strongly expressed in ventral part of the prosencephalon, the mesencephalon and neural tube. GLI3 expression was also expressed in otic vesicle. At day 40, the expression pattern was observed in pharyngeal archs and was restricted later at day 49 in maxillary and mandible. At the same time, GLI3 was also expressed in distal limb buds, floor plate of the telencephalon, diencephalon and mesencephalon, neural tube and strongly in kidney (Figure 4). 

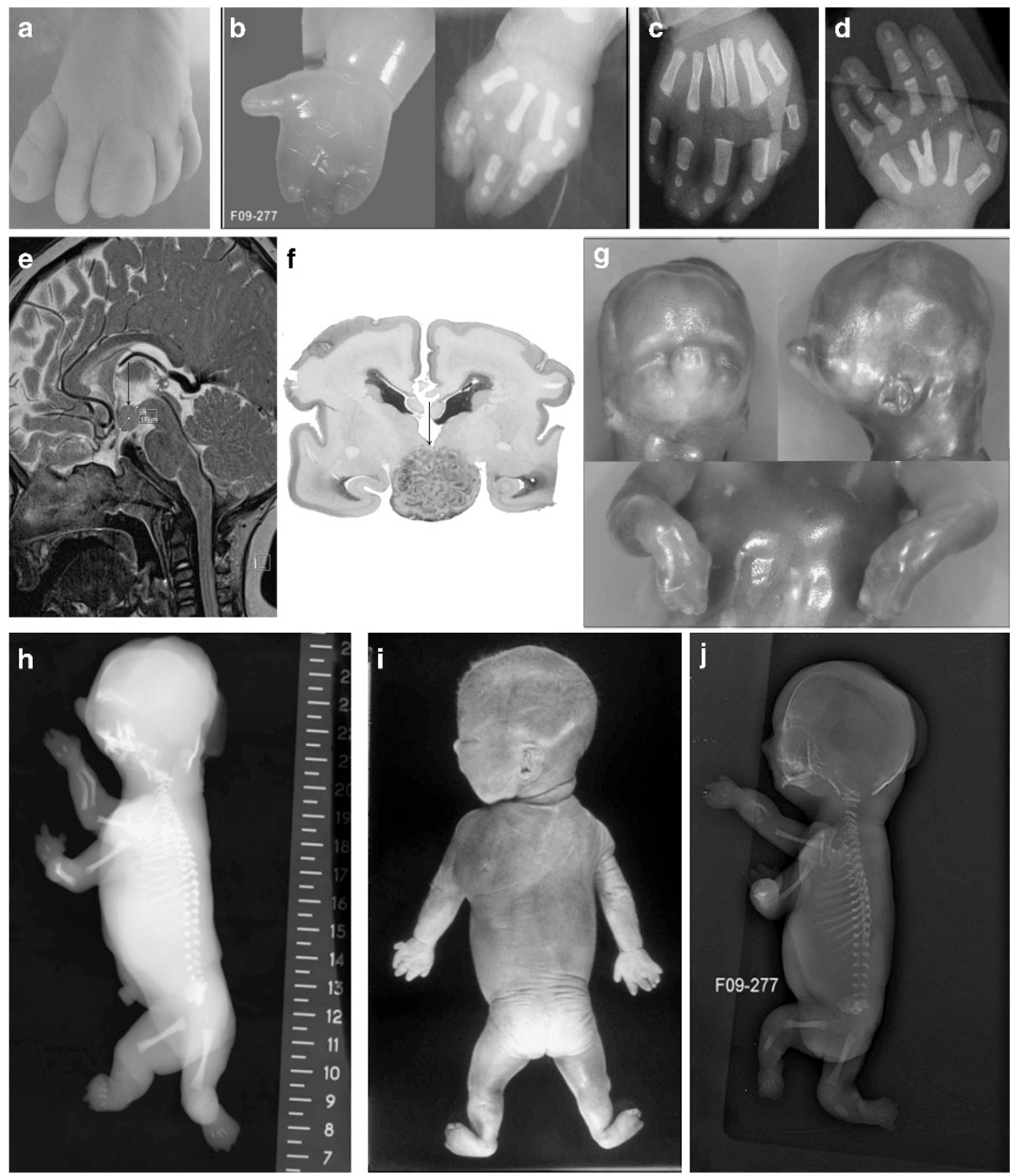

Figure 3 Photographs, radiographies and histological findings of PHS cases with identified GLI3 mutation. (a) Insertional PD and syndactyly (G072). (b) Oligodactyly (G080). (c) Insertional PD with a supernumerary metacarpal (G072). (d) Y-shaped metacarpal without PD (P15112). (e) Brain MRI showing a HH (P15112). (f) HH on neuropathological examination (G013). (g) Agnathia, hypoplastic maxillary, absence of oral orifice and oligosyndactyly (G012). (h) X-rays of G012 showing oligosyndactyly of hands and feet, arthrogryposis, mesomelia, bilateral radio-ulnar bowing, absence of tibia and fibula (G012). (i) (G024) and (j) (G080) showing micrognathia, micromelia, oligosyndactyly and club feet.

\section{DISCUSSION}

We present in this report molecular and clinical data of the second largest series of patients with GLI3 mutations. Molecular results of our series support previous genotype-phenotype correlations, showing that exonic deletions, missense mutations, as well as truncating variants localized outside the middle third of the GLI3 gene result in GCPS, while truncating mutations in the middle third result in PHS. Two truncating mutations in patients with preaxial PD mapped within the PHS region. Previous known exceptions to these correlations were described, for example, the recurrent c.2374C $>\mathrm{T}$ associated with a typical GCPS phenotype., ${ }^{911,19,20}$ These exceptions may be explained by a variable contribution of nonsense-mediated decay $^{12}$ or by the formation of unstable proteins with a very short half-life, effective nulls.

Only 10 GCPS probands of our series did fulfill all criteria suggested by Biesecker et $a l^{6}$ namely preaxial $\mathrm{PD}$, cutaneous syndactyly, widely spaced eyes and macrocephaly. Craniofacial features were absent or very subtle in 17 patients. Craniosynostosis was found in only two patients confirming the low-frequency association with GCPS. ${ }^{21}$ Along the same line, the diagnosis of PHS was confirmed in four individuals despite the absence of PD, whereas the clinical diagnostic criteria for PHS classically require the presence of insertional PD and a $\mathrm{HH}$ in the proband..$^{22}$

Interestingly, corpus callosum anomalies were found in nine patients, including seven patients with a truncating mutation located in the third end of GLI3. In the two previous series reported by Johnston et al, ${ }^{9,10}$ four GCPS patients with corpus callosum dysgenesis were also carrying a GLI3 truncating mutation lying in the C-terminal domain of the protein further confirming our finding that corpus callosum dysgenesis is fully part of GCPS spectrum and is mainly caused by terminal truncating mutations. Overlapping features with acrocallosal syndrome (ACLS, MIM\# 200990) associating callosal 

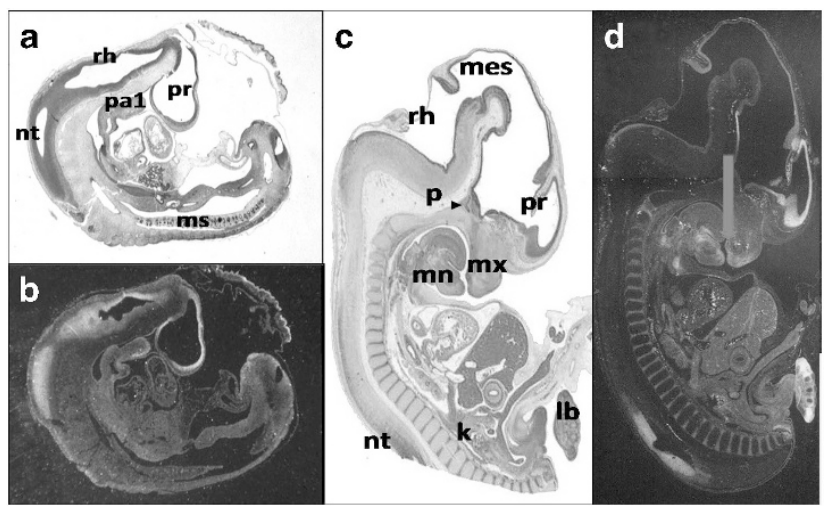

Figure 4 In situ hybridization of GL/3 during human development. (a, b) CS 15; (c, d) CS 19. (b, d) Slides hybridized with an antisense GL/3 probe. (a, c) Adjacent slides respectively to (b) and (d) stained with HES. In addition to the expression in central nervous system (prosencephalon (pr), rhombencephalon (rh) neural tube (nt)), limb bud (Ib), pituitary gland $(p$, arrowhead) and kidney (K), a signal was observed in human pharyngeal arches (pal) then in mandible ( $\mathrm{mn}$ ) and maxillary ( $\mathrm{mx}$ ) (red arrows). A full color version of this figure is available at the European Journal of Human Genetics journal online.

dysgenesis, hypertelorism, intellectual disability and $\mathrm{PD}^{23}$ are explained by an impaired GLI3 processing in patients with KIF7 mutations. ${ }^{24}$ Facial dysmorphism, as well as vermis dysgenesis with brainstem anomalies (molar tooth sign), strongly indicated the diagnosis of ACLS. Conversely, two GLI3 mutated cases with corpus callosum dysgenesis have been reported as ACLS ${ }^{25,26}$ and a third similar patient has been reported by Johnston et al. ${ }^{10}$ All three mutations were missense and clustered in the same region between aa 903 and 934 suggesting a potential severe phenotype associated with alterations of this region. Whatever, mutation analysis in both genes is therefore essential as the distinction between these two syndromes is of obvious significance for genetic counseling considering the difference in heredity and neurodevelopmental outcome and patients with a GLI3 mutation may be diagnosed as GCPS.

Interestingly, macrosomia was observed in at least 13\% of GCPS cases in our series. Macrosomia and PD are also observed in Simpson-Golabi-Behmel syndrome type 1 (MIM\# 312870), a $\mathrm{X}$-linked mental retardation syndrome ascribed to Glypican3 (GPC3) mutation, which was suspected in family G068, with two brothers displaying macrosomia and PD at birth. The frameshift GLI3 mutation was inherited from their asymptomatic father carrying a somatic mosaic mutation. Along the same way, we identified a mosaic large deletion in a GCPS patient with developmental delay (G059). To our knowledge, only one instance of GLI3 germline mosaicism has been already described in two PHS sibs, ${ }^{18}$ which is therefore a rare event.

Cerebral MRI may be useful to detect $\mathrm{HH}$ that was found in all PHS individuals of our series. Abnormal metacarpals in particular Y-shaped metacapals appear to be a more significant criterion than insertional PD. At least three PHS patients without PD were already reported. ${ }^{10,18,27}$ All of them presented fused or hypoplastic metacarpal. The mouse model for PHS Gli3 ${ }^{4699 / \Delta 699}$ displays abnormal metacarpal morphology with PD or oligodactyly at a lower frequency. ${ }^{28}$ Central poly/syndactyly and Y-shaped metacarpals are extremely uncommon in other syndromes. Although associated to a good neurodevelopmental outcome, PHS displays a wide range of severity varying from mild to lethal phenotypes depending on the severity of malformations present in the individuals, in particular bilateral kidney agenesis, craniofacial features (agnathia, absence of oral orifice, cleft palate or premaxillary agenesis in one case), heart defects and/or reductional limb defects. Interestingly, skeletal dysplasia with ulna bowing, tibia and fibula hypoplasia was already reported in other cases described as PHS, ${ }^{10,29,30}$ further suggesting that acromesomelic limb shortening with radio-ulnar bowing, tibial and fibular hypolasia/agenesis are part of the phenotypic spectrum of PHS. Interestingly, all mutations found in severe fetal phenotypes of our series were clustered in the middle third of the gene, between c.2941 and c.3324. To assess whether the severe craniofacial features observed (agnathia, absence of oral orifice) were a direct effect of the GLI3 mutation, we undertook the expression analysis of GLI3 during human development. Indeed, in addition to the early expression of GLI3 in pharyngeal and then later in mandible and maxillary, GLI3 was highly expressed in all target tissues of the disease.

Besides PHS cases, Y-shaped metacarpal is also observed in orofacio-digital syndrome type VI (OFD VI; MIM\# 277170). ${ }^{31}$ Overlap of PHS with OFD has been previously discussed as oral anomalies (oral frenula, hamartoma, cleft palate) and/or skeletal dysplasia are often associated to GLI3 mutations. ${ }^{10}$ Avila et al ${ }^{32}$ screened eight patients with OFD associated with midline abnormalities but no mutation was found. They suggested that GLI3 should be screened in patients with OFD only when associated to one of the pathognomonic sign of PHS ( $\mathrm{HH}$, mesoaxial PD, bifid epiglottis and IA).

In one case (G013), the association of IUGR, PD, bilateral renal agenesis and anal anteposition without macroscopic $\mathrm{HH}$ first led to the suspicion of Smith-Lemli-Opitz (SLO; MIM\# 270400). After exclusion of a cholesterol biosynthesis defect, a GLI3 screening identified a de novo frameshift mutation in exon 15. Retrospective analysis of the brain identified microscopic changes suggestive of hamartoma. This observation underlines the phenotypic overlap of PHS and SLO that was suggested previously, ${ }^{22}$ both disorders associating IUGR, PD and possible renal agenesis but IA, insertional PD and $\mathrm{HH}$ are exceptional in SLO. ${ }^{33}$

\section{CONCLUSION}

Here we report on clinical and molecular data of a large series of 76 individuals from 55 families carrying a heterozygous GLI3 mutation or rearrangement, 55 GCPS and 21 PHS, 41 being novel mutations. Our results render more precisely the genotype-phenotype correlation of GLI3 mutations proposed by Johnston et al, and further highlight the clinical overlap between GCPS and ACS and between PHS, SLO and OFD. Interestingly, our series including fetal cases enlarge the phenotypic spectrum of PHS to severe craniofacial and reductional limb defects, emphasize on the possible lethality of PHS with a clustering of truncating mutation in a subdomain of the 'PHS' GLI3 domain. In addition, we add CCA among frequent signs of GCPS with a strong genotype-phenotype correlation of corpus callosum dysgenesis with truncating $\mathrm{C}$ terminal mutations, and macrosomia as a new clinical feature of GCPS.

\section{CONFLICT OF INTEREST}

The authors declare no conflict of interest.

\section{ACKNOWLEDGEMENTS}

We are grateful to the patients and their parents participating in this study. 


\section{WEB SOURCES}

OMIM: http://www.ncbi.nlm.nih.gov/omim

PolyPhen2: http://genetics.bwh.harvard.edu/pph2

SIFT: http://blocks.fhcrc.org/sift/SIFT.html

Mutalyzer: http://www.humgen.nl/mutalyzer/1.0.1

LOVD: http://www.lovd.nl/3.0

1 Vortkamp A, Franz T, Gessler M, Grzeschik KH: Deletion of GLI3 supports the homology of the human Greig cephalopolysyndactyly syndrome (GCPS) and the mouse mutant extra toes (Xt). Mamm Genome Off J Int Mamm Genome Soc 1992; 3: 461-463.

2 Kang S, Rosenberg M, Ko VD, Biesecker LG: Gene structure and allelic expression assay of the human GLI3 gene. Hum Genet 1997; 101: 154-157.

3 Hall JG, Pallister PD, Clarren SK et al: Congenital hypothalamic hamartoblastoma, hypopituitarism, imperforate anus and postaxial polydactyly-a new syndrome? Part I: clinical, causal, and pathogenetic considerations. Am J Med Genet 1980; 7: 47-74.

4 Clarren SK, Alvord Jr EC, Hall JG: Congenital hypothalamic hamartoblastoma, $68(3)$ :hypopituitarism, imperforate anus, and postaxial polydactyly-a new syndrome? Part II: neuropathological considerations. Am J Med Genet 1980; 7: 75-83.

5 Biesecker L, Johnston J: Syndromic and non-syndromic GLI3 phenotypes. Clin Genet 2005; 68: 284-284.

6 Biesecker LG: The Greig cephalopolysyndactyly syndrome. Orphanet J Rare Dis 2008 3. 10 .

7 Johnston JJ, Walker RL, Davis S et al: Zoom-in comparative genomic hybridisation arrays for the characterisation of variable breakpoint contiguous gene syndromes. J Med Genet 2007; 44: e59.

8 Aza-Blanc P, Lin HY, Ruiz i Altaba A, Kornberg TB: Expression of the vertebrate Gli proteins in Drosophila reveals a distribution of activator and repressor activities. Development (Cambridge, England) 2000; 127: 4293-4301.

9 Johnston JJ, Olivos-Glander I, Killoran C et al: Molecular and clinical analyses of Greig cephalopolysyndactyly and Pallister-Hall syndromes: robust phenotype prediction from the type and position of GLI3 mutations. Am J Hum Genet 2005; 76: 609-622.

10 Johnston JJ, Sapp JC, Turner JT et al: Molecular analysis expands the spectrum of phenotypes associated with GLI3 mutations. Hum Mutat 2010; 31: 1142-1154.

11 Kalff-Suske M, Wild A, Topp J et al: Point mutations throughout the GLI3 gene cause Greig cephalopolysyndactyly syndrome. Hum Mol Genet 1999; 8: 1769-1777.

12 Furniss D, Critchley P, Giele H, Wilkie AOM: Nonsense-mediated decay and the molecular pathogenesis of mutations in SALL1 and GLI3. Am J Med Genet A 2007, 143A: 3150-3160.

13 Shin SH, Kogerman P, Lindström E, Toftgárd R, Biesecker LG: GLI3 mutations in human disorders mimic Drosophila cubitus interruptus protein functions and localization. Proc Natl Acad Sci USA 1999; 96: 2880-2884.

14 Krauss S, So J, Hambrock M et al: Point mutations in GLI3 lead to misregulation of its subcellular localization. PLoS One 2009; 4: e7471.
15 Wildeman M, van Ophuizen E, den Dunnen JT, Taschner PEM: Improving sequence variant descriptions in mutation databases and literature using the Mutalyzer sequence variation nomenclature checker. Hum Mutat 2008; 29: 6-13.

16 O'Rahilly R: Human embryo. Nature 1987; 329: 385.

17 Crosnier C, Attié-Bitach T, Encha-Razavi $F$ et al: JAGGED1 gene expression during human embryogenesis elucidates the wide phenotypic spectrum of Alagille syndrome. Hepatology (Baltimore, MD) 2000; 32: 574-581.

$18 \mathrm{Ng} \mathrm{D}$, Johnston JJ, Turner JT et al: Gonadal mosaicism in severe Pallister-Hall syndrome. Am J Med Genet A 2004; 124A: 296-302.

19 Debeer P, Peeters H, Driess S et al: Variable phenotype in Greig cephalopolysyndactyly syndrome: clinical and radiological findings in 4 independent families and 3 sporadic cases with identified GLI3 mutations. Am J Med Genet A 2003; 120A 49-58.

20 Furniss D, Kan S-H, Taylor IB et al: Genetic screening of 202 individuals with congenital limb malformations and requiring reconstructive surgery. J Med Genet 2009; 46: 730-735.

21 Hurst JA, Jenkins D, Vasudevan PC et al: Metopic and sagittal synostosis in Greig cephalopolysyndactyly syndrome: five cases with intragenic mutations or complete deletions of GLI3. Eur J Hum Genet 2011; 19: 757-762.

22 Biesecker LG, Graham Jr JM: Pallister-Hall syndrome. J Med Genet 1996; 33: 585-589.

23 Putoux A, Nampoothiri S, Laurent $\mathrm{N}$ et al: Novel KIF7 mutations extend the phenotypic spectrum of acrocallosal syndrome. J Med Genet 2012; 49: 713-720.

24 Putoux A, Thomas S, Coene KLM et al: KIF7 mutations cause fetal hydrolethalus and acrocallosal syndromes. Nat Genet 2011; 43: 601-606.

25 Elson E, Perveen R, Donnai D, Wall S, Black GCM: De novo GLI3 mutation in acrocallosal syndrome: broadening the phenotypic spectrum of GLI3 defects and overlap with murine models. J Med Genet 2002; 39: 804-806.

26 Speksnijder L, Cohen-Overbeek TE, MFCM Knapen et al: A de novo GLI3 mutation in a patient with acrocallosal syndrome. Am J Med Genet A 2013; 161: 1394-1400.

27 Verloes A, David A, Ngô L, Bottani A: Stringent delineation of Pallister-Hall syndrome in two long surviving patients: importance of radiological anomalies of the hands. J Med Genet 1995; 32: 605-611.

28 Hill P, Wang B, Rüther U: The molecular basis of Pallister-Hall associated polydactyly. Hum Mol Genet 2007; 16: 2089-2096.

29 Encha-Razavi F, Larroche JC, Roume J et al: Congenital hypothalamic hamartoma syndrome: nosological discussion and minimum diagnostic criteria of a possibly familial form. Am J Med Genet 1992; 42: 44-50.

30 Roscioli T, Kennedy D, Cui J et al: Pallister-Hall syndrome: unreported skeletal features of a GLI3 mutation. Am J Med Genet A 2005; 136A: 390-394.

31 Poretti A, Vitiello G, Hennekam RCM et al: Delineation and diagnostic criteria of Oral-Facial-Digital Syndrome type VI. Orphanet J Rare Dis 2012; 7: 4.

32 Avila M, Gigot N, Aral B et al: GLI3 is rarely implicated in OFD syndromes with midline abnormalities. Hum Mutat 2011; 32: 1332-1333.

33 Quélin C, Loget $\mathrm{P}$, Verloes A et al: Phenotypic spectrum of fetal Smith-Lemli-Opitz syndrome. Eur J Med Genet 2012; 55: 81-90.

34 Ruppert JM, Vogelstein B, Arheden K, Kinzler KW: GLI3 encodes a 190-kilodalton protein with multiple regions of GLI similarity. Mol Cell Biol 1990; 10: 5408-5415. 